\title{
Antihypertensive Effect of Radix Paeoniae Alba in Spontaneously Hypertensive Rats and Excessive Alcohol Intake and High Fat Diet Induced Hypertensive Rats
}

\author{
Chen Su-Hong, ${ }^{1,2}$ Chen Qi, ${ }^{3}$ Li Bo, ${ }^{1}$ Gao Jian-Li, ${ }^{1}$ Su Jie, ${ }^{1}$ and Lv Gui-Yuan ${ }^{1,2}$ \\ ${ }^{1}$ Zhejiang Chinese Medical University, Hangzhou, Zhejiang 310053, China \\ ${ }^{2}$ Wenzhou Medical University, Wenzhou, Zhejiang 325035, China \\ ${ }^{3}$ The Third People's Hospital of Hangzhou, Hangzhou 310009, China \\ Correspondence should be addressed to Gao Jian-Li; jianligaozcmu@foxmail.com and Lv Gui-Yuan; lv.gy@263.net
}

Received 18 September 2014; Revised 21 January 2015; Accepted 22 January 2015

Academic Editor: Chee Y. Choo

Copyright ( 2015 Chen Su-Hong et al. This is an open access article distributed under the Creative Commons Attribution License, which permits unrestricted use, distribution, and reproduction in any medium, provided the original work is properly cited.

\begin{abstract}
Radix Paeoniae Alba (Baishao, RPA) has long been used in traditional Chinese medicine formulation to treat hypertension by repression the hyperfunction of liver. However, whether the RPA itself has the antihypertensive effect or not is seldom studied. This study was to evaluate the protective effect of RPA on hypertensive rats. Alcohol in conjunction with a high fat diet- (ACHFD-) induced hypertensive rats and spontaneously hypertensive rats (SHR) was constantly received either RPA extract ( $25 \mathrm{or} 75 \mathrm{mg} / \mathrm{kg}$ ) or captopril $(15 \mathrm{mg} / \mathrm{kg})$ all along the experiments. As a result, RPA extract $(75 \mathrm{mg} / \mathrm{kg})$ could significantly reduce systolic blood pressure of both ACHFD-induced hypertensive rats and SHR after 9-week or 4-week treatment. In ACHFD-induced hypertensive rats, the blood pressure was significantly increased and the lipid profiles in serum including triglyceride, total cholesterol, LDLcholesterol, and HDL-cholesterol were significantly deteriorated. Also, hepatic damage was manifested by a significant increase in alanine transaminase (ALT) and aspartate transaminase (AST) in serum. The RPA extract significantly reversed these parameters, which revealed that it could alleviate the liver damage of rats. In SHR, our result suggested that the antihypertensive active of RPA extract may be related to its effect on regulating serum nitric oxide (NO) and endothelin (ET) levels.
\end{abstract}

\section{Introduction}

China is undergoing remarkable economic development, social reform, and globalization with rapidly changing lifestyles in recent years, which has resulted in alarming prevalence of hypertension. Hypertension and prehypertension were significantly associated with increased all-cause and cardiovascular mortality $(P<0.0001)$. In 2005, 2.33 million (95\% CI 2.21-2.45) cardiovascular deaths were attributable to increased blood pressure in China [1]. Worldwide, the number of adults with hypertension in 2025 is predicted to increase by about $60 \%$ to a total of 1.56 billion [2]. The factors contributing to the development of hypertension are various, and dietary habit undoubtedly is an important one.

The association between alcohol consumption, blood pressure, and the prevalence of hypertension has been studied in the past 20 years. The relationship between light-tomoderate alcohol consumption (1-2 standard wine drinks a day) and the incident of hypertension remains controversial [3], but excessive alcohol intake dose elevates blood pressure, with global estimates that the attributable risk for hypertensive disease from alcohol is $16 \%$ [4]. In the UK, the accepted alcohol limits are 16 grams a day for women-the alcohol in just over a medium glass of wine-and 32 grams for men, roughly half a bottle of red wine.

Recent epidemiological studies further reveal that the high-fat diet is also strongly associated with the development and aggravation of hypertension and chronic target organ damage $[5,6]$. In addition, it has been reported that combined consumption of a high fat diet with alcohol can aggravate obesity and liver fat accumulation [7, 8].

It is a tradition that herbal medicine, such as Radix Paeoniae Alba (RPA), Chrysanthemum, and Uncaria rhynchophylla, is used in the treatment of hypertension in China. Among them, RPA is the major constituent material of many 
complex preparations (mixture formulation of herbal drugs) to treat hypertension [9]. RPA, the dry root of Paeonia lactiflora Pall, is composed of nearly 10 types of medicinal substances, mainly paeoniflorin. According to the scientific research, RPA had wide variety of pharmacological actions such as anti-inflammatory, antioxidation, and strengthening immunity [10-12]. Qian et al. found that paeoniflorin, the main active ingredient in the root of Paeonia Radix, inhibited the proliferation of pulmonary artery smooth muscle cells (PASMCs) by blocking cell cycle progression in the $\mathrm{S}$ phase through activation of A2BAR under hypoxic conditions [13]. Ethanol extract of Paeonia lactiflora Pall relaxes vascular smooth muscle via endothelium-dependent and Akt- and SOCE-eNOS-cGMP-mediated pathways through activation of both $\mathrm{K}(\mathrm{Ca})$ and $\mathrm{K}(\mathrm{ATP})$ channels and inhibition of L-type $\mathrm{Ca}^{2+}$ channels [14].

To further clarify the antihypertensive mechanisms of RPA, the present study was designed to investigate the antihypertensive effect of RPA, both in the alcohol in conjunction with a high fat diet- (ACHFD-) induced hypertension model and spontaneously hypertensive rat (SHR) model. Furthermore, we also investigate the relationship between its protection effect in ACHFD induced liver damage and its antihypertensive effect.

\section{Materials and Methods}

2.1. Chemicals. Alcoholic drink (Alcohol $=52 \%$, Red Star Corp, Beijing, China) was diluted with distilled water to alcohol $=23 \%(\mathrm{v} / \mathrm{v})$. Biochemical reagents, such as total cholesterol (TC), triglyceride (TG), high density lipoproteincholesterol (HDL-C), low density lipoprotein-cholesterol (LDL-C), alanine transaminases (ALT), and aspartate transaminases (AST), were purchased from MeiKang Chemical Co. (Ningbo, Zhejiang, China). ELISA kits, such as plasma renin activity (PRA), angiotensin II (Ang II), aldosterone (ALD), and endothelin-1 (ET-1), were all purchased from HengYuan Chemical Co. (Shanghai, China). Nitric oxide (NO) kit was purchased from Nanjing Technology Co., Ltd. (JiangSu, China). The ethanol extract of RPA was obtained from Zelang Medical Technolody Co. (Nanjing, Jiangsu, China). The paeoniflorin in the extract was $53 \%$ detected by HPLC analysis.

2.2. Animal Groups. Male SD rats weighing 190-220 g were obtained from Animal Supply Center of Zhejiang Academy of Medical Science (Hangzhou, China). Male normotensive Wistar Kyoto (WKY) and spontaneously hypertensive rats (SHR) weighing 300-350 g were obtained from Vital River Laboratory Animal Inc. (Beijing, China). All the animals were housed in standard environmental conditions at a temperature of $25 \pm 1^{\circ} \mathrm{C}$, humidity of $55 \pm 5 \%$, and a $12 / 12 \mathrm{~h}$ light/dark cycle and allowed a standard pellet chow diet and water ad libitum for 1 week. All procedures were performed according to protocols following the guidelines for the Use and Care of Laboratory Animals published by the Zhejiang province (2009).
2.3. Animal Treatments. SD rats were firstly divided into 5 groups $(n=7)$. The normal group received a standard pellet chow diet and water throughout the first 8 weeks of the experiment. The ACHFD-induced hypertensive rats, given high fat diet and 23\% alcohol-water drinking for 8 weeks, have a $\geq 15 \%$ increase in systolic blood pressure (SBP). Daily water consumption was estimated individually for every animal 1 week before the experiment. During the experiment, water consumption was controlled. This protocol was adopted based on our preliminary studies [15]. For the next 8 weeks, group 2 rats were set as the model control group and continued to receive a high fat diet and alcohol; group 3 rats were given a high fat diet, alcohol, and captopril; groups 4 and 5 rats were given a high fat diet, alcohol, and RPA extract (at the doses of 25 and $75 \mathrm{mg} / \mathrm{kg}$, p.o.). SHRs were randomly assigned to four groups of eight rats each. The first group was set as SHR control group; the second group received captopril; Groups 3 and 4 received RPA extract (at the doses of 25 and $75 \mathrm{mg} / \mathrm{kg}$, p.o.). Throughout the experiment, body weight was evaluated.

2.4. Blood Pressure Measurement. Blood pressure, which included SBP, diastolic blood pressure (DBP), and mean arterial blood pressure (MBP), was measured by a noninvasive method of tail-cuff plethysmography (Shanghai Alcott Biotech Co., Ltd., Shanghai China) every week. To get accurate blood pressure, at least 6 consecutive determinations were recorded.

2.5. Biochemical Analysis. At the end of experiment, the ACHFD-induced hypertensive rats were fasted overnight and anesthetized with pentobarbital. Blood was drawn from the ophthalmic venous plexus and centrifuged at $3000 \mathrm{rpm}$ for $10 \mathrm{~min}$. The concentrations of TC [16], TG, LDL-C, and HDL$\mathrm{C}$ were determined by the ACCUTE (TBA-40FR) automatic biochemical analyzer (Toshiba, Japan). The activities of ALT and AST were also determined by TBA-40FR using the method of IFCC [17].

2.6. Determination of PRA, Ang II, ALD, and ET-1 Activities. At the end of experiment, the SHR were fasted overnight and anesthetized with pentobarbital. Blood was drawn from the ophthalmic venous plexus and centrifuged at $3000 \mathrm{rpm}$ for $10 \mathrm{~min}$. Serum was separated to determine plasma renin activity (PRA), angiotensin II (Ang II), aldosterone (ALD), and endothelin-1 (ET-1) activities by the method of enzyme linked immunosorbent assay [18]. All of the procedures were performed as described in the assay kit. Briefly, the various samples for ELISA measurement were diluted in $0.1 \%$ BSA/Tris-buffered saline and were incubated in a 96-well plate precoated with a capture antibody directed against PRA, Ang II, ALD, or ET-1 for $30 \mathrm{~min}$ at $37^{\circ} \mathrm{C}$. The wells were then washed five times in $0.05 \%$ Tween $20 / \mathrm{PBS}$ and incubated with a secondary antibody against PRA, Ang II, ALD, or ET-1 conjugated to horseradish peroxidase for an additional $30 \mathrm{~min}$ at $37^{\circ} \mathrm{C}$. The plates were then washed again five times, substrate solution containing $\mathrm{H}_{2} \mathrm{O}_{2}$ and tetramethylbenzidine (TMB) was added, and optical density was determined at $450 \mathrm{~nm}$. All assays were done in duplicate, and the protein levels 
were calculated using a standard curve derived from known concentrations of the respective recombinant proteins.

2.7. Determination of Serum NO Level. NO has a brief halflife and is rapidly converted to the stable end-products $\mathrm{NO}_{2}{ }^{-}$ and nitrate $\left(\mathrm{NO}_{3}{ }^{-}\right)$in typical oxygenated aqueous solutions [19]. Therefore, the serum NO level was evaluated by nitrate reductase method [20]. Blood sample was centrifuged at $3000 \mathrm{rpm}$ for $10 \mathrm{~min}$ and serum was separated to determine $\mathrm{NO}$ concentration. The reductase nitrate restores all $\mathrm{NO}_{3}{ }^{-}$to $\mathrm{NO}_{2}{ }^{-}$, which can be determinate by the excellent colorimetric reagent to measure total nitrite as an indicator of $\mathrm{NO}$ production as described before. All of the procedures were performed as described in the assay kit.

2.8. Statistical Analysis. All measurements were expressed as the mean \pm standard deviation and subjected to oneway analysis of variance (ANOVA), followed by Fisher's least significant difference (LSD) comparison. $P$ value of $<0.05$ was considered statistically significant. All analyses were performed using an updated version of SPSS software.

\section{Results}

3.1. Effects of Radix Paeoniae Alba Extract on Body Weight. The effects of the RPA extract on the weight of ACHFDinduced hypertensive rats $(n=7)$ or SHR $(n=8)$ were compared with those of rats fed a control diet (Table 1). We found that PRA did not affect the body weight of the rats in both hypertensive models. Although it has been reported that excessive alcohol consumption combined with high fat diet results in weight gain and metabolic disorders [21, 22], the different result from our experiment may be explained by anorexia phenomenon of the high fat rats and the empty calories caused by alcohol consumption [7].

\subsection{Effects of Radix Paeoniae Alba Extract Supplement on} Blood Pressure of ACHFD-Induced Hypertensive Rats. Before the experiment, there were no significant differences in SBP, DBP, and MBP among the groups. During the period of model establishment, SBP, DBP, and MBP of the normal group (NC) were constant while the administration of alcohol and high fat diet significantly increased the SBP, DBP, and MBP of all the model groups (Figure 1(a)). The RPA extract groups presented a downtrend line, which is meaningful. After 9 weeks of treatment with RPA, the SBP of the RPA high dose group (PH) was reduced from $162 \mathrm{mmHg}$ to $141 \mathrm{mmHg}$ and was significant lower than that of the model control group (MC). On the other hand, only captopril could significant reduce SBP, DBP, and MBP of ACHFD-induced hypertensive rats after 7-week treatment (Figures 1(b) and 1(c)).

\subsection{Effects of Radix Paeoniae Alba Extract Supplement on} Lipid Profile. As shown in Table 2 the concentrations of TC and LDL-cholesterol were significantly higher while the concentration of HDL-cholesterol was significantly lower in the rats fed with high fat diet and alcohol, compared with the normal diet group. This morbid state was significantly prevented by the treatment with the plant extract at the dose of $75 \mathrm{mg} / \mathrm{kg}$. At the same dose, the increase of atherogenic index was also prevented as compared to untreated rats. The circulating concentration of TG was not changed significantly by the high fat diet and alcohol consumption.

3.4. Effects of Radix Paeoniae Alba Extract Supplement on Liver Function. The effects of RPA extract on liver function in ACHFD-induced hypertensive rats $(n=7)$ or SHR $(n=$ 8) are summarized in Table 2. Chronic alcohol and high fat administration resulted in a greatly increased AST and ALT concentration in serum, as compared to normal rats. Upon RPA (low dose) treatment, levels of ALT and AST significantly decreased as compared to untreated hypertensive rats.

3.5. Effects of Radix Paeoniae Alba Extract Supplement on Blood Pressure of the SHR. Changes in SBP, DBP, and MBP of the SHR during 4 weeks of administration are shown in Figure 2. Before treatment, SBP, DBP, and MBP were significantly $(P<0.01)$ higher in the SHR control than in the WKY control. Since the third week of treatment, there was a significant suppression of SBP, DBP, and MBP $(P<0.01)$ in $\mathrm{PH}$-treated SHR compared to the SHR control. In the fourth week, the SBP of the RPA high dose group (PH) was reduced from $208 \mathrm{mmHg}$ to $200 \mathrm{mmHg}$ and was significantly lower than that of the model control group (MC). The positive control drug, captopril, also significantly lowered SBP, DBP, and MBP in the SHR.

3.6. Effects of Radix Paeoniae Alba Extract Supplement on Renin-Angiotensin-Aldosterone System (RAAS) Activities. The results of plasma renin activity (PRA), angiotensin II (Ang II), and aldosterone (ALD) activity analysis after feeding for 4 weeks are shown in Figure 3. As a result, RPA extract treatment caused a significant decrease in serum ALD levels but presented no significant influence in the Ang II and PRA activity in the serum.

3.7. Effects of Radix Paeoniae Alba Extract Supplement on NO and ET-1 Concentration. The serum nitric oxide (NO) and endothelin-1 (ET-1) measurements are presented in Figure 3. The serum NO concentration in the treatment groups was significantly higher than that in the SHR control group. This indicates that the oral administration of RPA extract increased the serum NO concentration. Also, the addition of this herbal extract markedly suppressed the increased concentration of serum ET-1 concentration.

\section{Discussion}

In traditional Chinese medicine, the overactive liver function is 240 recognized as a major cause of hypertension, and Radix Paeoniae Alba could have an antihypertensive effect by repressing the hyperfunction of liver. In this study, we observed antihypertensive properties of the RPA extract in two different models of hypertension (spontaneous or alcohol and high fat diet-induced), its mechanism may be related to the lipid lowering, liver protection, and endothelial function improvement. 
TABLe 1: Effect of Radix Paeoniae Alba extract on the body weight of rats.

\begin{tabular}{|c|c|c|c|c|}
\hline Group & Parameter & Initial weight (g) & Final weight $(\mathrm{g})$ & Weight gain $(\mathrm{g})$ \\
\hline \multirow{5}{*}{ ACHFD rats $(n=7)$} & & $245.50 \pm 15.69$ & $423.00 \pm 26.05$ & $178.38 \pm 26.05$ \\
\hline & control & $246.10 \pm 19.47$ & $427.00 \pm 12.18$ & $181.75 \pm 19.59$ \\
\hline & cap & $237.40 \pm 8.06$ & $426.00 \pm 23.71$ & $189.43 \pm 21.02$ \\
\hline & $\mathrm{PH}$ & $243.90 \pm 13.98$ & $420.67 \pm 38.50$ & $175.78 \pm 38.32$ \\
\hline & $\mathrm{PL}$ & $244.30 \pm 9.57$ & $417.00 \pm 28.52$ & $170.78 \pm 33.40$ \\
\hline \multicolumn{2}{|c|}{ WKY normal $(n=8)$} & $328.00 \pm 24.54$ & $343.13 \pm 23.50$ & $15.13 \pm 28.80$ \\
\hline \multicolumn{2}{|c|}{ SHR control $(n=8)$} & $335.88 \pm 23.13$ & $333.25 \pm 16.20$ & $-2.63 \pm 20.58$ \\
\hline \multicolumn{2}{|c|}{ SHR cap $(n=8)$} & $329.25 \pm 24.50$ & $349.25 \pm 32.88$ & $20.00 \pm 14.73$ \\
\hline \multicolumn{2}{|c|}{ SHR PH $(n=8)$} & $332.38 \pm 24.85$ & $342.50 \pm 27.32$ & $10.13 \pm 30.65$ \\
\hline \multicolumn{2}{|c|}{ SHR PL $(n=8)$} & $342.63 \pm 27.24$ & $353.50 \pm 29.17$ & $10.88 \pm 20.41$ \\
\hline
\end{tabular}

ACHFD: Alcohol in conjunction with a high fat diet; cap: captopril; PH: RPA extract high dose group (75 mg/kg); PL: RPA extract low dose group (25 mg/kg).

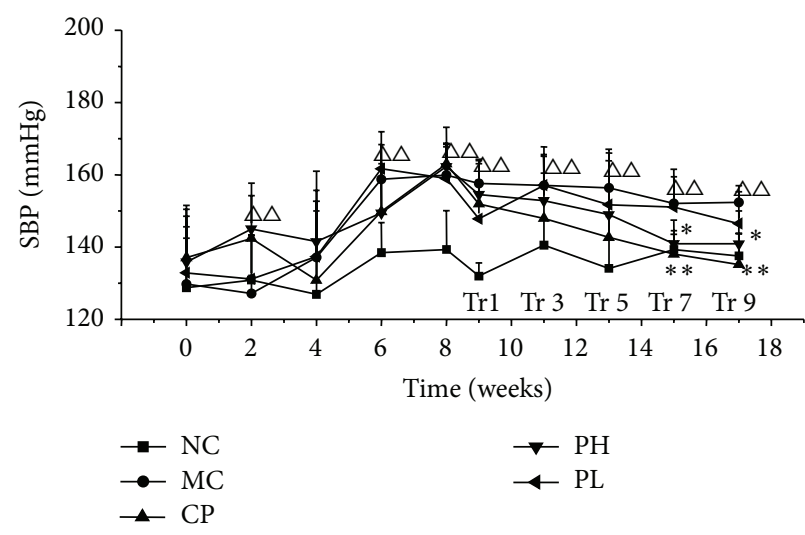

(a)

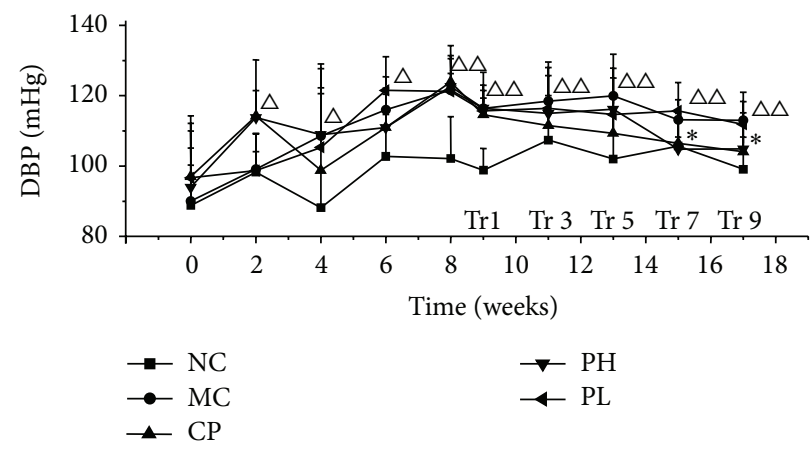

(b)

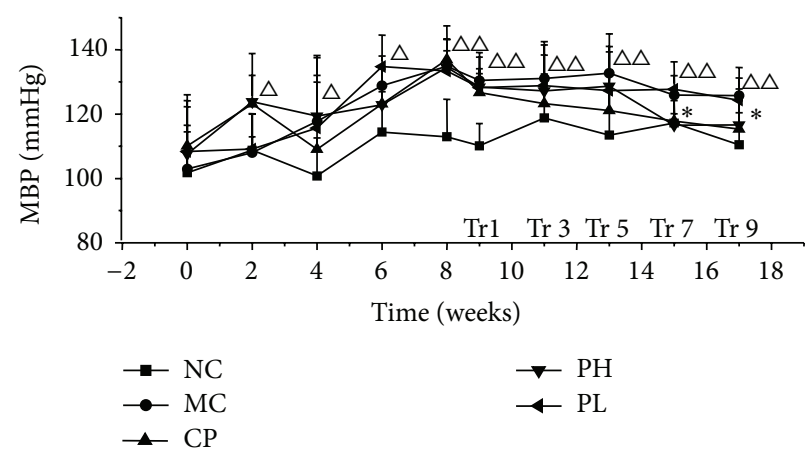

(c)

FIGURE 1: SBP, DBP, and MBP changed in ACHFD-induced hypertensive rat model establishment period (0-8 week) or treatment period (917 week). Values are expressed as the mean $\pm \mathrm{SD}(n=7) .\left({ }^{\triangle} P<0.05,{ }^{\triangle} P<0.01\right.$ versus normal control, ${ }^{*} P<0.05,{ }^{* *} P<0.01$ versus model group.) SBP: systolic blood pressure; DBP: diastolic blood pressure; MBP: mean blood pressure; NC: normal control group; CP: captopril group; MC: model control group; PH: RPA extract high dose group (75 mg/kg); PL: RPA extract low dose group (25 mg/kg); Tr: treatment.

Obesity, diet, and alcohol consumption constitute major environmental determinations of blood pressure elevation [23]. In the present study, alcohol and high fat feeding significantly increased total cholesterol, LDL-cholesterol, and the atherogenic index and decreased HDL-cholesterol. It followed by the blood vessel damage caused by fat deposits [24]. Chronic alcohol drinking with high-fat consumption led to the abnormal lipid metabolism and liver damage, as well as increase of SBP in rats. Therefore, we strongly propose that long term consumption of alcohol with a high fat diet could produce an appropriate model for recent excessive nutritional and alcohol abuse.

In this study, RPA extract $(75 \mathrm{mg} / \mathrm{kg})$ significantly prevented the increase of total cholesterol, LDL-cholesterol, atherogenic index, and the decrease of HDL-cholesterol and also significantly prevented the abnormal drop of HDL-cholesterol/TC ratio. HDL-cholesterol/TC ratio is a superior measure of cardiovascular diseases risk than 


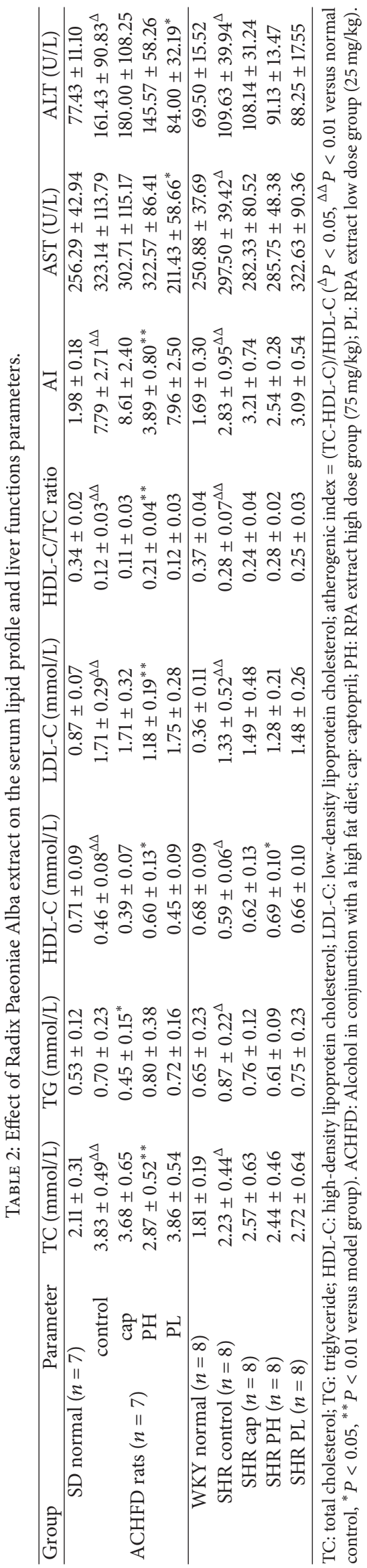



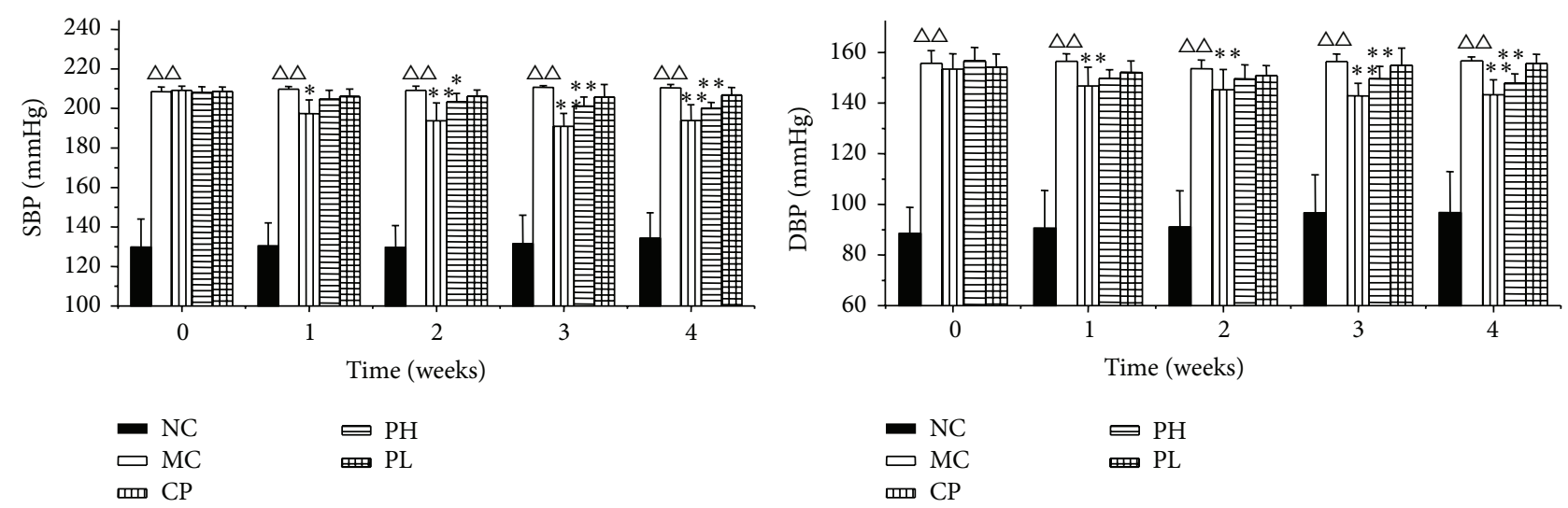

(a)

(b)

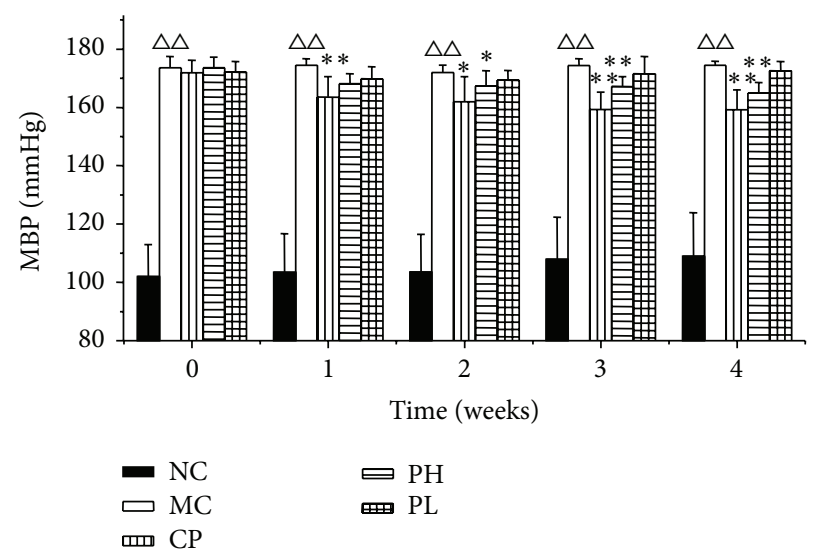

(c)

FIGURE 2: SBP, DBP, and MBP during 4 weeks of treatment in the SHR. Values are expressed as the mean \pm SD $(n=8) .\left({ }^{\triangle} P<0.05,{ }^{\triangle} P<<0.01\right.$ versus normal control, ${ }^{*} P<0.05,{ }^{* *} P<0.01$ versus model group.) SBP: systolic blood pressure; DBP: diastolic blood pressure; MBP: mean blood pressure; NC: normal group; CP: captopril group; MC: model group; PH: RPA extract high dose group (75 mg/kg); PL: RPA extract low dose group $(25 \mathrm{mg} / \mathrm{kg})$.

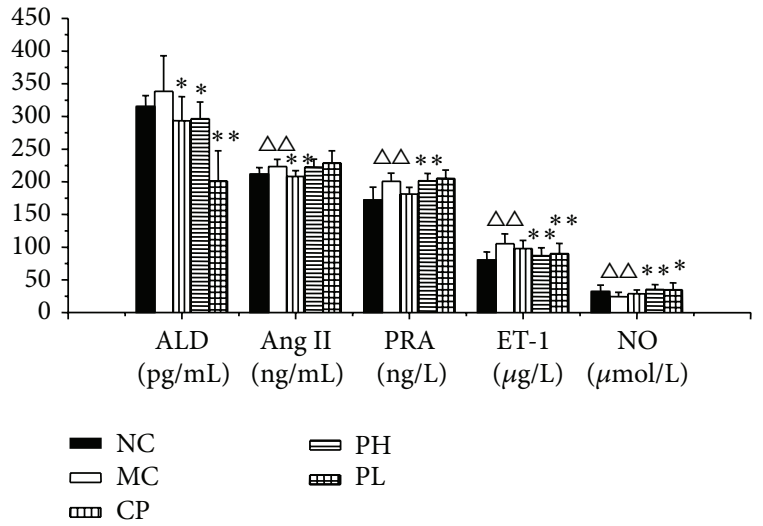

Figure 3: Serum PRA, Ang II, ALD, NO, and ET-1 concentration after four weeks of treatment. Values are expressed as the mean \pm SD $(n=8) .{ }^{\triangle} P<0.05,{ }^{\triangle} \triangle P<0.01$ versus normal control, ${ }^{*} P<0.05$, ${ }^{* *} P<0.01$ versus model group.) NC: normal control group; CP: captopril group; MC: model control group; PH: Radix Paeoniae Alba extract high dose group (75 mg/kg); PL: Radix Paeoniae Alba extract low dose group $(25 \mathrm{mg} / \mathrm{kg})$.
non-HDL-cholesterol. It captures the protective effect of HDL-cholesterol as well as the harmful effects of non-HDLcholesterol in a single parameter and remains one of the best dyslipidaemic measures [25]. Moreover, significant increases in the serum levels of ALT and AST are conventional indicators of liver damage [26]. Administration of the RPA extract tended to attenuate the increased concentrations of serum ALT and AST.

Several experiments have linked alcohol ingestion, high fat diet, and hypertension via a mechanism of elevated tissue angiotensin II levels, depletion of the endothelial nitric oxide generating system, and overproduction of endothelin$1[27,28]$. NO dilates blood vessel and inhibits platelet aggregation and proliferation of vascular smooth muscle cells [29]. The decrease of NO concentration in the blood may help explain the vascular pathology of hypertension. Based on the fact that angiotensin II increases eNOS expression and endothelial NO production by activating the angiotensin II type 2 receptor [30], Kleinhenz et al. suggested that ethanolinduced increases in the activity of the renin-angiotensin system contribute to enhanced vascular eNOS expression 
and NO production [31]. The link between NO availability and the metabolic adaptation to a high fat diet was also studied by Razny et al. [28]. They suggested that increased NO availability attenuates some high fat diet induced alterations in metabolism. In our study of spontaneously hypertensive rats, the serum concentration of NO in the normotensive controls was significantly higher than that in the hypertensive controls. The administration of RPA extract significantly increased serum NO concentration. However, there was no difference in serum Ang II concentration among the groups.

In contrast to NO, ET-1 is a powerful vasoconstrictor that has been demonstrated to mediate increased blood pressure. ET-1 exerts its effects through the activation of $\mathrm{ET}_{\mathrm{A}}$ and $\mathrm{ET}_{\mathrm{B}}$ receptors $[32,33] . \mathrm{ET}_{\mathrm{A}}$ receptors are present on vascular smooth muscle cells, whereas $\mathrm{ET}_{\mathrm{B}}$ receptors are located predominantly on endothelial cells. $\mathrm{ET}_{\mathrm{A}}$ receptor activation results in vasoconstriction and smooth muscle proliferation, whereas $\mathrm{ET}_{\mathrm{B}}$ receptor binding stimulates nitric oxide $(\mathrm{NO})$ synthesis, resulting in vasodilation [34]. In a model of visceral obesity and hypertension, da Silva et al. find that a longterm high fat diet may cause visceral obesity and increased arterial pressure and ET-1 appears to play an important role in the maintenance of arterial pressure [35]. In our study, RPA extract significantly inhibited the abnormal increase of the serum ET-1 level in spontaneous hypertension rats. Combined with previous results, we suggest that RPA extract might decrease the higher endothelin-1 levels and stimulated the $\mathrm{ET}_{\mathrm{B}}$ receptors which finally resulted in the massive release of NO.

The ancient literature Kai Bao Ben Cao has mentioned the potential vasoprotective properties of Radix Paeoniae Alba (Baishao). Here, our present study proves that the antihypertension effect of RPA may result from its liver protection activity and its improvement of endothelial function by reducing ET-1 and increasing NO concentrations.

\section{Conflict of Interests}

The authors declare that they have no conflict of interests.

\section{Authors' Contribution}

Chen Su-Hong and Chen Qi contributed equally to this work and should be considered co-first authors.

\section{Acknowledgments}

This work was supported by China National Natural Science Foundation (81274123 to GY L, 81374003 to SH C), Zhejiang Provincial Program for the Cultivation of High-level Innovative Health Talents to SH C, and the Zhejiang Provincial Key Laboratory Project (no. 2012E10002 to GY L).

\section{References}

[1] J. He, D. Gu, J. Chen et al., "Premature deaths attributable to blood pressure in China: a prospective cohort study," The Lancet, vol. 374, no. 9703, pp. 1765-1772, 2009.
[2] J. Chen, "Epidemiology of hypertension and chronic kidney disease in China," Current Opinion in Nephrology and Hypertension, vol. 19, no. 3, pp. 278-82, 2010.

[3] L. J. Beilin, I. B. Puddey, and V. Burke, "Alcohol and hypertension-kill or cure?” Journal of Human Hypertension, vol. 10, supplement 2, pp. S1-S5, 1996.

[4] I. B. Puddey and L. J. Beilin, "Alcohol is bad for blood pressure," Clinical and Experimental Pharmacology and Physiology, vol. 33, no. 9, pp. 847-852, 2006.

[5] R. G. Morrison, C. Mills, A. L. Moran et al., "A moderately high fat diet promotes salt-sensitive hypertension in obese Zucker rats by impairing nitric oxide production," Clinical and Experimental Hypertension, vol. 29, no. 6, pp. 369-381, 2007.

[6] S. Chung, C. W. Park, S. J. Shin et al., “Tempol or candesartan prevents high-fat diet-induced hypertension and renal damage in spontaneously hypertensive rats," Nephrology Dialysis Transplantation, vol. 25, no. 2, pp. 389-399, 2010.

[7] I. S. Lee, S. Park, K. Park, and R. Choue, "Hepatoprotective activity of scutellariae radix extract in mice fed a high fat diet with chronic alcohol exposure," Phytotherapy Research, vol. 25, no. 9, pp. 1348-1353, 2011.

[8] J. Ren, "Interaction between high-fat diet and alcohol dehydrogenase on ethanol-elicited cardiac depression in murine myocytes," Obesity, vol. 15, no. 12, pp. 2932-2941, 2007.

[9] R. Feng and L. Y. Zhen, "Effects of total glucosides of paeong on improving insulin sensitivity,lowering blood pressure and enhancing antioxidation in metabolic syndrome-hypertensive rats," Chinese Clinical Pharmacology, vol. 15, pp. 978-981, 2010.

[10] Y. Bao, C. Jing, and W. Shi, "Effects of Chinese herbal recipes on immunity in immunosuppressive mice.," African Journal of Traditional, Complementary, and Alternative Medicines, vol. 9, no. 4, pp. 548-552, 2012.

[11] Y.-F. Li, M. Wang, X.-Y. Wang et al., "Pharmacokinetic properties of albiflorin and paeoniflorin after oral administration of pure compound, Radix Paeoniae alba extract and DangguiShaoyao-San extract to rats," Journal of Asian Natural Products Research, vol. 13, no. 2, pp. 117-127, 2011.

[12] L. Y. Zhen, J. J. Pan, and J. H. Lv, "Pharmacological effect in plants of Radix Paeoniae Alba," Guangzhou Pharmacy, vol. 4, pp. 66-68, 2011.

[13] G. Qian, J. Cao, C. Chen et al., "Paeoniflorin inhibits pulmonary artery smooth muscle cells proliferation via upregulating $A_{2 B}$ adenosine receptor in rat," PLoS ONE, vol. 8, no. 7, Article ID e69141, 2013.

[14] S. N. Jin, J. F. Wen, T. T. Wang, D. G. Kang, H. S. Lee, and K. W. Cho, "Vasodilatory effects of ethanol extract of Radix Paeoniae Rubra and its mechanism of action in the rat aorta," Journal of Ethnopharmacology, vol. 142, no. 1, pp. 188-193, 2012.

[15] X. Hu, B. Li, S. S. Lei, J. Su, G. Y. Lv, and S. H. Chen, "Effect of three unhealthy lifestyle factors on blood pressure, blood biochemistry and microcirculation of rats," Journal of Zhejiang Chinese Medical University, vol. 38, no. 5, pp. 608-612, 2014.

[16] J. Abele and H. Khayam-Bashi, "Aqueous primary standard for use in measuring cholesterol by the cholesterol oxidase method," Clinical Chemistry, vol. 25, no. 1, pp. 132-135, 1979.

[17] T. Gordon, W. P. Castelli, M. C. Hjortland, W. B. Kannel, and T. R. Dawber, "High density lipoprotein as a protective factor against coronary heart disease. The Framingham study," The American Journal of Medicine, vol. 62, no. 5, pp. 707-714, 1977.

[18] E. Engvall and P. Perlmann, "Enzyme-linked immunosorbent assay (ELISA) quantitative assay of immunoglobulin G," Immunochemistry, vol. 8, no. 9, pp. 871-874, 1971. 
[19] S. Doganay, C. Evereklioglu, H. Er et al., "Comparison of serum NO, TNF- $\alpha$, IL- $1 \beta$, sIL-2R, IL- 6 and IL- 8 levels with grades of retinopathy in patients with diabetes mellitus," Eye, vol. 16, no. 2, pp. 163-170, 2002.

[20] G. Giovannoni, J. M. Land, G. Keir, E. J. Thompson, and S. J. R. Heales, "Adaptation of the nitrate reductase and Griess reaction methods for the measurement of serum nitrate plus nitrite levels," Annals of Clinical Biochemistry, vol. 34, no. 2, pp. 193-198, 1997.

[21] J. S. Tolstrup, J. Halkjær, B. L. Heitmann et al., "Alcohol drinking frequency in relation to subsequent changes in waist circumference," The American Journal of Clinical Nutrition, vol. 87, no. 4, pp. 957-963, 2008.

[22] H. Schröder, J. A. Morales-Molina, S. Bermejo et al., "Relationship of abdominal obesity with alcohol consumption at population scale," European Journal of Nutrition, vol. 46, no. 7, pp. 369-376, 2007.

[23] L. J. Beilin, "Diet, alcohol and hypertension," Clinical and Experimental Hypertension. Part A Theory and Practice, vol. 11, no. 5-6, pp. 991-1010, 1989.

[24] R. Stocker and J. F. Keaney Jr., "Role of oxidative modifications in atherosclerosis," Physiological Reviews, vol. 84, no. 4, pp. 13811478, 2004.

[25] R. J. Stevens, R. L. Coleman, B. L. Shine, and R. R. Holman, "Could non-HDL cholesterol replace total/HDL cholesterol ratio to estimate coronary heart disease risk in the UKPDS risk engine?" Diabetologia, vol. 47, supplement 1, p. A61, 2004.

[26] G. S. Achliya, S. G. Wadodkar, and A. K. Dorle, "Evaluation of hepatoprotective effect of Amalkadi Ghrita against carbon tetrachloride-induced hepatic damage in rats," Journal of Ethnopharmacology, vol. 90, no. 2-3, pp. 229-232, 2004.

[27] K. Husain, M. Vazquez, R. A. Ansari, M. P. Malafa, and J. Lalla, "Chronic alcohol-induced oxidative endothelial injury relates to angiotensin II levels in the rat," Molecular and Cellular Biochemistry, vol. 307, no. 1-2, pp. 51-58, 2008.

[28] U. Razny, B. Kiec-Wilk, L. Wator et al., "Increased nitric oxide availability attenuates high fat diet metabolic alterations and gene expression associated with insulin resistance," Cardiovascular Diabetology, vol. 10, article 68, 2011.

[29] R. G. Knowles and S. Moncada, "Nitric oxide synthases in mammals," Biochemical Journal, vol. 298, part 2, pp. 249-258, 1994.

[30] S. Olson, R. Oeckler, X. Li et al., "Angiotensin II stimulates nitric oxide production in pulmonary artery endothelium via the type 2 receptor," The American Journal of Physiology - Lung Cellular and Molecular Physiology, vol. 287, no. 3, pp. L559-L568, 2004.

[31] D. J. Kleinhenz, R. L. Sutliff, J. A. Polikandriotis et al., "Chronic ethanol ingestion increases aortic endothelial nitric oxide synthase expression and nitric oxide production in the rat," Alcoholism: Clinical and Experimental Research, vol. 32, no. 1, pp. 148-154, 2008.

[32] T. Sakurai, M. Yanagisawa, Y. Takuwa et al., "Cloning of a cDNA encoding a non-isopeptide-selective subtype of the endothelin receptor," Nature, vol. 348, no. 6303, pp. 732-735, 1990.

[33] H. Arai, S. Hori, I. Aramori, H. Ohkubo, and S. Nakanishi, "Cloning and expression of a cDNA encoding an endothelin receptor," Nature, vol. 348, no. 6303, pp. 730-732, 1990.

[34] A. Nohria, L. Garrett, W. Johnson, S. Kinlay, P. Ganz, and M. A. Creager, "Endothelin-1 and vascular tone in subjects with atherogenic risk factors," Hypertension, vol. 42, no. 1, pp. 43-48, 2003.
[35] A. A. da Silva, J. J. Kuo, L. S. Tallam, and J. E. Hall, "Role of endothelin-1 in blood pressure regulation in a rat model of visceral obesity and hypertension," Hypertension, vol. 43, no. 2, pp. 383-387, 2004. 


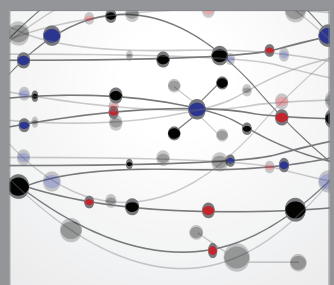

The Scientific World Journal
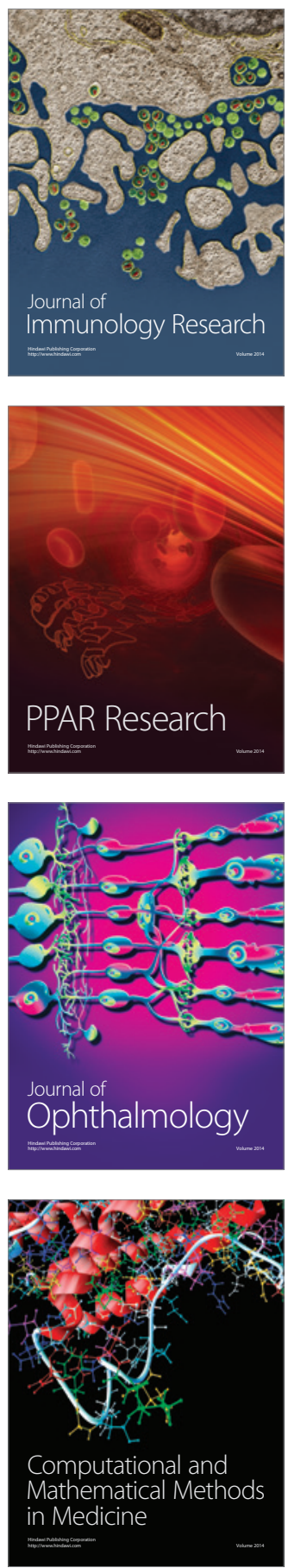

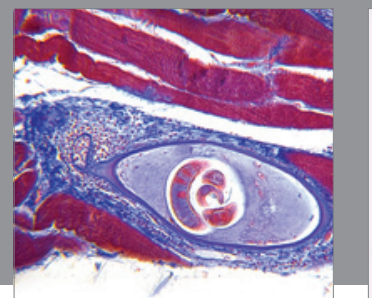

Gastroenterology

Research and Practice
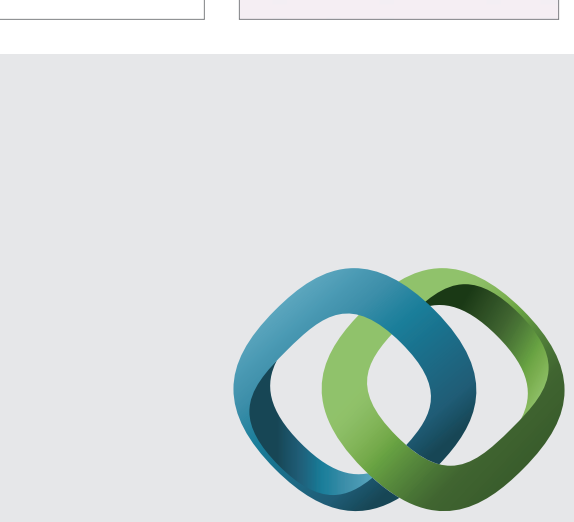

\section{Hindawi}

Submit your manuscripts at

http://www.hindawi.com
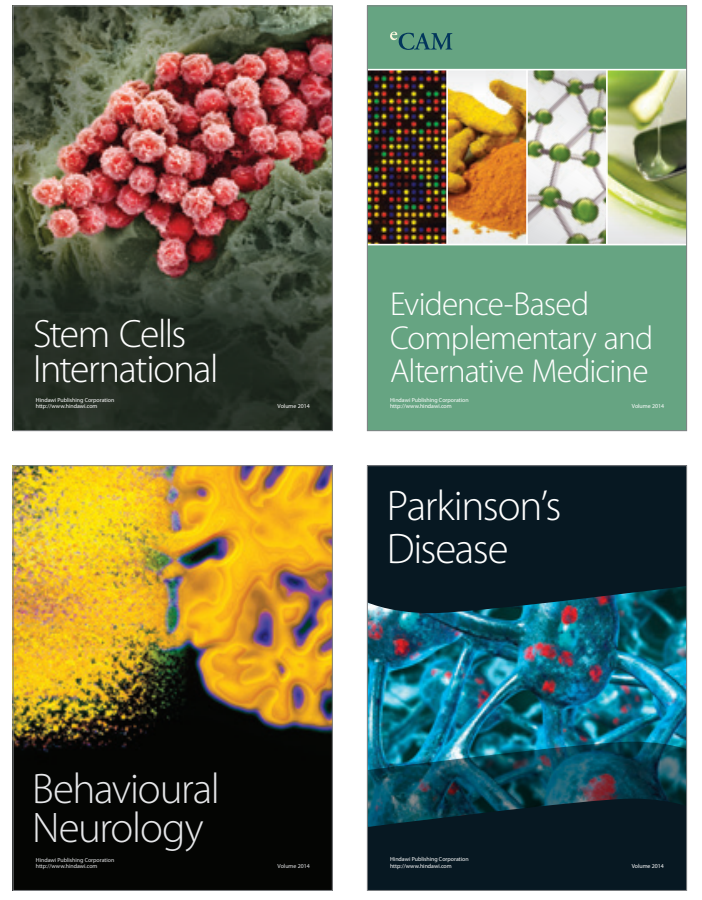
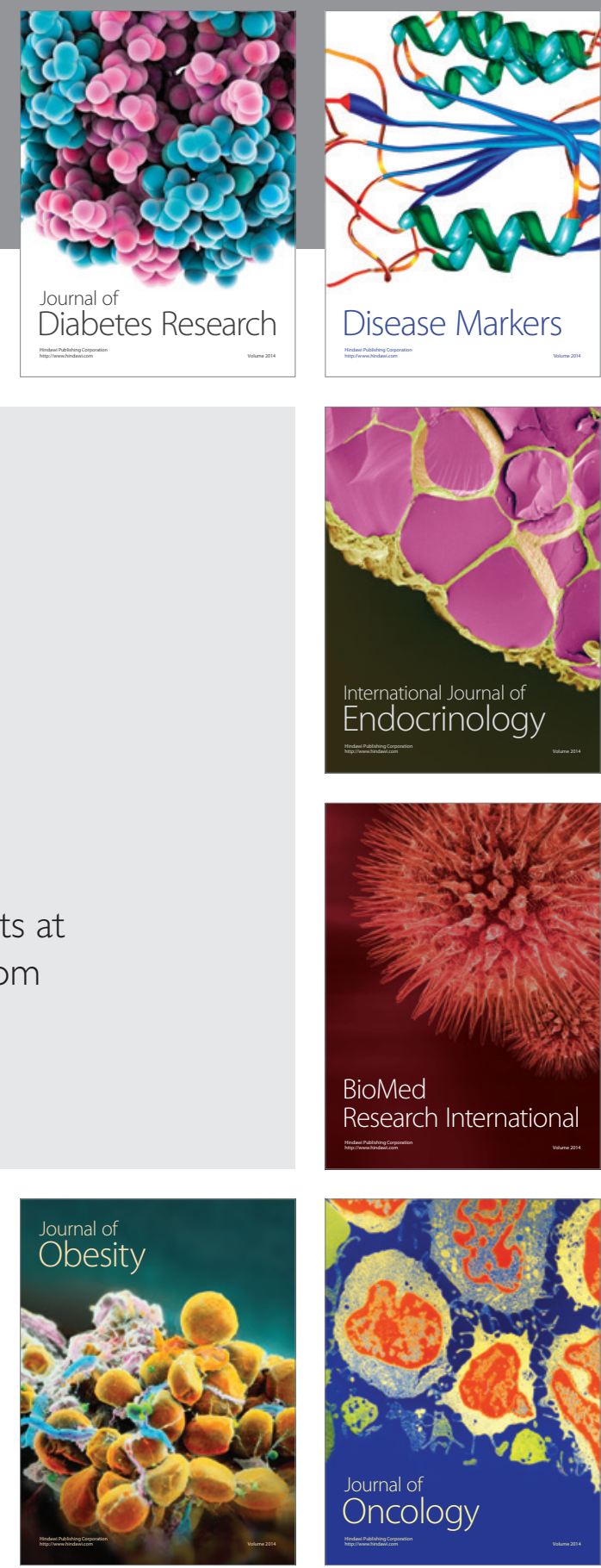

Disease Markers
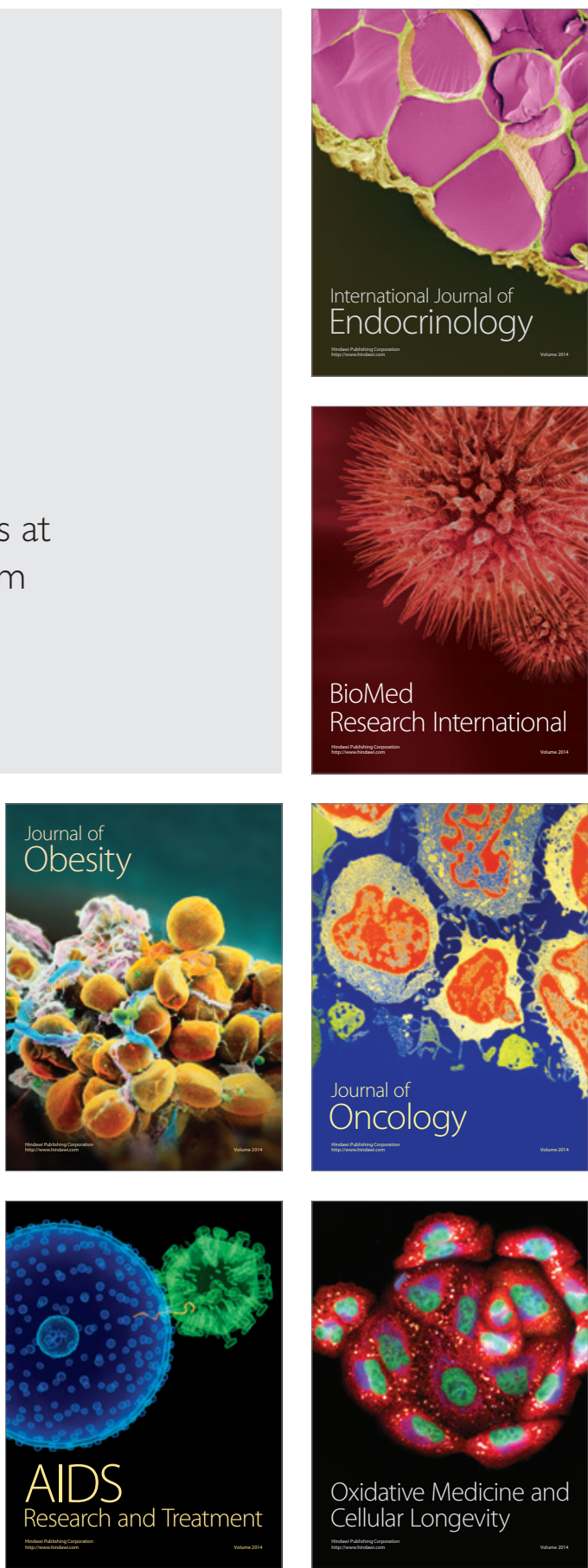